Zbl Arbeitsmed 2016 - 66:220-223

DOI 10.1007/s40664-016-0119-3

Online publiziert: 21. Juni 2016

๑) Springer-Verlag Berlin Heidelberg 2016

CrossMark

\section{Erratum zu: \\ Zbl Arbeitsmed (2016) 66:92-94 \\ doi: $10.1007 / \mathrm{s} 40664-016-0090-\mathrm{z}$}

Bedauerlicherweise wurde in Ausgabe 2/2016 des Zentralblatts für Arbeitsmedizin, Arbeitsschutz und Ergonomie nicht die überarbeitete Version des Beitrags „Poliomyelitis. Aktuelle Aspekte im arbeitsmedizinischen Kontext" von Schöffel et al. publiziert.

Wir bitten, dies zu entschuldigen und die aktuelle Version zu beachten.

\section{Definition}

Poliomyelitis (PM) ist eine akute virale Infektionskrankheit des zentralen Nervensystems (ZNS) durch das Poliovirus $(\mathrm{PV})$. Die Bezeichnung „Kinderlähmung" entstand, da häufig Klein- und Schulkinder an der PM-Erkrankte [3, 5].

\section{Epidemiologie}

Im 19. und 20. Jahrhundert kam es in den USA sowie Ländern Mittel- und Nordeuropas zu epidemischen Ausbreitungen. Die Inzidenz der PM erreichte 1916 in den Vereinigten Staaten ihren Höhepunkt (41/100.000 Erkrankte/ Einwohner) [3, 4]. Durch Einführung der Schutzimpfung konnte die Inzidenz seit 1955 drastisch gesenkt werden. Das 1988 von der Weltgesundheitsorganisation (WHO) ins Leben gerufene Projekt der globalen Polioeradikation

Diese Arbeit ist Bestandteil eines Promotionsverfahrens von Frau U. Drews.

Die Online-Version des Originalartikels ist unter doi:10.1007/s40664-016-0090-zzu finden.

N. Schöffel • U. Drews · M. H. K. Bendels · D. Brüggmann · D. A. Groneberg

Institut für Arbeitsmedizin, Sozialmedizin und Umweltmedizin, Goethe-Universität Frankfurt, Frankfurt am Main, Deutschland

\title{
Erratum zu: Poliomyelitis
}

\section{Aktuelle Aspekte im arbeitsmedizinischen Kontext}

erzielte eine weitere, deutliche Reduktion $[3,4]$. Es wird derzeit weltweit von ca. 1500-2000 jährlichen Neuerkrankungen ausgegangen. In Deutschland wurde die letzte, durch einen Wildtypusvirus hervorgerufene PM 1990 registriert [2, $3,10]$.

\section{Übertragungsmechanismen}

Der enge Kontakt mit einer infizierten Person bildet den primären Infektionsweg. Dabei sind 2 Übertragungsmechanismen möglich [2, 3, 7]:

1. Fäkal-oraler Weg: Aufnahme über den Mund in den Verdauungstrakt über kontaminierte Finger oder Objekte,

2. Aerogene Übertragung: Theoretisch möglich ist auch eine Übertragung aufgrund der primären Vermehrung des PV in den Pharynxepithelien. Diese ist jedoch aus arbeitsmedizinischer Sicht nicht relevant.

\section{Morphologie des Poliovirus}

Das PV ist ein Mitglied der Familie der Picornaviren. Das PV ist ein umweltstabiles Virus (hohe Resistenz gegen $\mathrm{pH}$ Schwankungen, niedrige Temperaturen oder Alkohol). Formaldehyd, Temperaturen über $50{ }^{\circ} \mathrm{C}$ und UV-Licht führen jedoch zur schnellen Inaktivierung. Das PV besitzt 3 Serotypen (PV1-3), welche alle eine PM hervorrufen können [3, 12, 14].

\section{Zellzyklus}

Die Vermehrung des PV findet im Zytoplasma der Wirtszelle statt. Dies wird initiiert durch das Eindringen des Virus in die Wirtszelle und folgender Bindung an einen Rezeptor (CD-155). Nach der zellulären Aufnahme wird die virale RNA freigesetzt und translatiert. Die Replikation der RNA wird durch die virale RNAPolymerase ermöglicht. Die virale RNA und Strukturproteine werden zu einem Virion zusammengesetzt und nach Lyse der infizierten Zelle freigesetzt $[7,11]$.

\section{Symptomatik}

Die Inkubationszeit beträgt 2-35 Tage. Die meisten Infektionen (> $95 \%$ ) verlaufen jedoch asymptomatisch. Bei ca. $5 \%$ der Infizierten manifestiert sich die PM in 3 klinischen Formen: abortive, nichtparalytische und paralytische PM. Die abortive PM kennzeichnet sich durch uncharakteristische Symptome wie Fieber, Übelkeit und Myalgien. Sie heilt in ca. $75 \%$ der Fälle folgenlos aus. In 1-2 \% der Fälle entwickelt sich durch Befall des ZNS eine nichtparalytische PM (aseptische Meningitis). Für die nichtparalytische PM bzw. aseptische Meningitis ist die Manifestation von Fieber, Nackensteifigkeit, Rückenschmerzen und Muskelspasmen 3-7 Tage nach Präsenz der abortiven PM charakteristisch. Nach einem oder mehreren Tagen ist ein Übergang vom nichtparalytischen Manifestationsbild zur paralytischen PM möglich. Dies zeigt sich bei ca. 0,1-1\% der Infizierten. Eine Rückbildung der Lähmungen kann in den nächsten Wochen und Monaten erfolgen. Nach mehr als 12 Monaten ist dies jedoch eine Rarität [3, 4, 7]. 


\section{Post-Polio-Syndrom}

Das Post-Polio-Syndrom (PPS) ist eine neurologische Störung, die als Spätkomplikation nach einer paralytischen PM auftritt. Charakteristisch ist eine progrediente Muskelschwäche, die auf den kontinuierlichen Abbau der noch funktionsfähigen Motoneurone zurückgeführt wird [1].

\section{Diagnostik}

Bei klinischer Präsentation eines Patienten mit akut einsetzender, schlaffer Muskelparalyse ohne Sensibilitätsausfälle bei abgeschwächten oder nicht vorhandenen Sehnenreflexen kann die Diagnose einer paralytischen PM klinisch gestellt werden. Zur diagnostischen Sicherung der Infektion wird der Erreger mittels Zellkulturen isoliert. Als Materialien hierfür eignen sich eine Stuhlprobe, ein Rachenabstrich oder -spülwasser wie auch eine Liquorprobe. Das PV kann über Antikörpernachweis mittels Komplementbindungsreaktion (KBR), mittels der Polymerase-Kettenreaktion (PCR) und über einen Neutralisationstest (NT) mit Antiseren aus Zellkultur erfolgen [3, 9].

\section{Therapie}

Da eine kausale Therapie nicht möglich ist, muss die Behandlung symptomatisch erfolgen. Physiotherapie sowie entsprechende orthopädische Hilfsmittel sind grundlegende Bestandteile der Therapie und Versorgung. Bei Lähmung des Zwerchfalles kann eine Unterstützung der Atmung mit entsprechenden Geräten sowie Tracheotomie notwendig sein $[3,7,11]$.

\section{Impfung}

Bis zur Entwicklung der Polioimpfung im Jahre 1954 von Jonas Salk erkrankten 500.000-1,2 Mio. Personen pro Jahr an einer PM. Heutzutage stehen zur Prävention 2 Arten von Impfungen zur Verfügung: Ein inaktivierter Impfstoff (IPV), der hauptsächlich in den Industrieländern eingesetzt wird und eine orale Poliovakzine (OPV) mit Lebend-

Zbl Arbeitsmed 2016 66:220-223 DOI 10.1007/s40664-016-0119-3

(c) Springer-Verlag Berlin Heidelberg 2016

N. Schöffel · U. Drews · M. H. K. Bendels · D. Brüggmann · D. A. Groneberg

Erratum zu: Poliomyelitis. Aktuelle Aspekte im arbeitsmedizinischen Kontext

Zusammenfassung

Poliomyelitis (PM) ist eine virale Infektionskrankheit durch das Poliovirus. Die Bezeichnung "Kinderlähmung" entstand, da häufig Klein- und Schulkinder an der PM erkrankten. Die meisten Infektionen (> $95 \%$ ) verlaufen asymptomatisch. 0,1-1\% entwickeln eine Paralyse, die jedoch teilweise vollständig reversibel ist. Zur Diagnostik werden Proben aus Rachenspülwasser, Stuhl, Blut oder Liquor herangezogen. Mittels serologischem Antikörpernachweis (ELISA) oder PCR kann dann der direkte wie indirekte
Nachweis der Infektion erfolgen. Seit 1954 gibt es eine präventive Schutzimpfung, die im Laufe der Jahre jedoch eine Anpassung erfahren hat. Diese Übersicht beinhaltet die aktuellen Erkenntnisse zum Thema PM unter besonderer Berücksichtigung arbeitsmedizinischer Aspekte.

Schlüsselwörter

Poliovirus · Picornaviren · Schutzimpfung . Meldepflicht · Prognose

\section{Erratum to: Poliomyelitis. Current aspects in occupational medical issues}

\section{Abstract}

Poliomyelitis (PM) is an infectious disease caused by the poliovirus (PV). The term infantile paralysis is based on the virus' propensity to affect children. About $95 \%$ of all people who are affected by the virus are asymptomatic. Only $0.1-1 \%$ of all infected people show a certain degree of paralysis, from which most people recover completely. Diagnosis is usually made from a stool sample, a swab of the pharynx or cerebrospinal fluid via the detection of PV antigen by enzyme immunoassay or polymerase chain reaction. The first polio vaccine was developed in the 1954 by Jonas Salk. This review outlines the current knowledge about PM, with particular attention given to occupational issues.

Keywords

Poliomyelitis - Picornaviruses - Vaccination . Mandatory disease notification $\cdot$ Prognosis impfstoff, die in Entwicklungsländern Verwendung findet. Das primär entwickelte orale Polio-Vakzin (OPV) mit einem Polio-Lebendimpfstoff ist verantwortlich für sogenannte „Vakzineassoziierte paralytische PoliomyelitisErkrankungen“ (VAPP), die 1- bis 2-mal jährlich durch Rückmutation des Virus beobachtet werden können $[4,6,8,10]$. Das Auftreten dieser Komplikation veranlasste die STIKO im Jahr 1998 dazu, die OPV nicht mehr zu empfehlen und stattdessen durch die IPV ersetzen zu lassen. Aktuell gelten folgende Empfehlungen der STIKO (• Tab. 1; [13-15]): Zum Schutz vor der Poliomyelitis sollte ein $\mathrm{zu}$ injizierender Impfstoff, inaktivierte Polio-Vakzine (IPV), eingesetzt werden (ggf. als Kombinationsimpfstoff). Im Alter von 9-17 Jahren wird für Jugendliche eine Auffrischimpfung mit einem Impfstoff, der IPV enthält, empfohlen. Eine mit OPV begonnene Grundimmunisierung wird mit IPV komplettiert.

\section{Meldepflicht}

Seit dem 01. 01. 2001 ist das Infektionsschutzgesetz (IfSG) gültig. Nach diesem ist bei Verdacht, Erkrankung oder Tod an PM als auch bei indirektem oder direktem Erregernachweis einer akuten Infektion eine namentliche Meldung an das Gesundheitsamt vorzunehmen. Nach einer jeweiligen Überprüfung erfolgt vom Gesundheitsamt eine Übermittlung der Informationen an die zuständige Landesbehörde, welche darauffolgend das Robert Koch-Institut (RKI) informiert [7, $11,13]$. 
Tab. 1 Standardimpfungen des Erwachsenenalters, Indikations- und Auffrischimpfungen - hier Polio (adaptiert nach [15])

\begin{tabular}{|c|c|c|}
\hline Kat & Indikation & Anwendungshinweise \\
\hline S/A & $\begin{array}{l}\text { Alle Personen bei fehlender oder un- } \\
\text { vollständiger Grundimmunisierung } \\
\text { Alle Personen ohne einmalige Auf- } \\
\text { frischimpfung }\end{array}$ & $\begin{array}{l}\text { Erwachsene, die im Säuglings- und Kleinkindal- } \\
\text { ter eine vollständige Grundimmunisierung und } \\
\text { im Jugendalter oder später mindestens eine } \\
\text { Auffrischimpfung erhalten haben oder die als } \\
\text { Erwachsene nach Angaben in den Fachinfor- } \\
\text { mationen grundimmunisiert wurden und eine } \\
\text { Auffrischimpfung erhalten haben, gelten als } \\
\text { vollständig immunisiert } \\
\text { Ausstehende Impfungen werden entsprechend } \\
\text { den Angaben in den Fachinformationen mit IPV } \\
\text { nachgeholt } \\
\text { Darüber hinaus wird eine routinemäßige Auf- } \\
\text { frischimpfung für Erwachsene nicht empfohlen }\end{array}$ \\
\hline 1 & $\begin{array}{l}\text { Für folgende Personengruppen ist eine } \\
\text { Impfung indiziert: } \\
\text { - Reisende in Regionen mit Infekti- } \\
\text { onsrisiko (die aktuelle epidemische } \\
\text { Situation ist zu beachten, insbeson- } \\
\text { dere die Meldungen der WHO) } \\
\text { - Aussiedler, Flüchtlinge und Asylbe- } \\
\text { werber, die in Gemeinschaftsunter- } \\
\text { künften leben, bei der Einreise aus } \\
\text { Gebieten mit Poliomyelitis-Risiko, }^{\text {siehe }}{ }^{\text {a }}\end{array}$ & $\begin{array}{l}\text { Ausstehende Impfungen der Grundimmuni- } \\
\text { sierung bzw. eine nicht dokumentierte Grund- } \\
\text { immunisierung werden mit IPV nachgeholt } \\
\text { bzw. es erfolgt eine Auffrischimpfung, wenn die } \\
\text { letzte Impfung länger als } 10 \text { Jahre zurückliegt } \\
\text { Personen ohne Nachweis einer Grundimmu- } \\
\text { nisierung sollten vor Reisebeginn wenigstens } \\
2 \text { Impfstoffdosen IPV erhalten }\end{array}$ \\
\hline B & $\begin{array}{l}\text { - Personal der oben genannten Ein- } \\
\text { richtungen } \\
\text { - medizinisches Personal, das engen } \\
\text { Kontakt zu Erkrankten haben kann } \\
\text { - Personal in Laboren mit Poliomyeli- } \\
\text { tis-Risiko }\end{array}$ & $\begin{array}{l}\text { Ausstehende Impfungen der Grundimmunisie- } \\
\text { rung werden mit IPV nachgeholt bzw. es erfolgt } \\
\text { eine Auffrischimpfung, wenn die letzte Imp- } \\
\text { fung der Grundimmunisierung bzw. die letzte } \\
\text { Auffrischimpfung länger als } 10 \text { Jahre zurückliegt }\end{array}$ \\
\hline
\end{tabular}

ampfempfehlungen für Aussiedler, Flüchtlinge oder Asylbewerber in Gemeinschaftsunterkünften. Es wird empfohlen, Schutzimpfungen bei Bewohnern von Gemeinschaftsunterkünften möglichst frühzeitig durch den Öffentlichen Gesundheitsdienst (ÖGD) oder durch vom ÖGD beauftragte Ärzte zumindest zu beginnen. Die Vervollständigung der Grundimmunisierung sollte nach dem Verlassen der Gemeinschaftsunterkünfte durch die am späteren Aufenthaltsort niedergelassenen Ärzte oder durch den ÖGD erfolgen. Vorliegende Impfdokumentationen sollten nach Möglichkeit berücksichtigt werden; die Empfehlungen der STIKO sollten dem Vorgehen zugrunde gelegt werden. Bei ungeimpften Erwachsenen bzw. Erwachsenen mit unklarem Impfstatus sollten Impfungen gegen Diphtherie und Tetanus, gegen Poliomyelitis sowie bei seronegativen Personen gegen Hepatitis B durchgeführt werden. Erwachsene sollen die nächste fällige Td-Impfung (Auffrischimpfung) einmalig als Tdap-Kombinationsimpfung erhalten. Nach 1970 Geborene sollten einmalig gegen Masern (MMR) geimpft werden. Frauen im gebärfähigen Alter sollten zweimal gegen Röteln (MMR) geimpft werden und seronegative Frauen mit Kinderwunsch sollten zweimal gegen Varizellen geimpft werden. Bei ungeimpften Kindern bzw. Kindern mit unklaren Impfstatus sollten Impfungen gegen Diphtherie, Tetanus und Pertussis sowie gegen Poliomyelitis, Masern, Mumps, Röteln, Varizellen und gegen Hepatitis B, Meningokokken C und HPV (nur bei Mädchen), bei Säuglingen und Kleinkindern auch gegen Rotaviren, Haemophilus influenzae Typ b und Pneumokokken durchgeführt werden.

\section{Maßnahmen bei Kontakt}

Bei klinischem oder labordiagnostischem Verdacht auf eine PM sind eine unverzügliche Einweisung ins Krankenhaus sowie ein Aufenthalt unter Isolierungsmaßnahmen und rigiden Hygienevorkehrungen anzustreben, solange bis der labordiagnostische Nachweis einer Polioinfektion negativ ausfällt. Im Sinne der Stuhldiagnostik sollte unverzüglich eine Probenabgabe an das RKI und das Nationale Referenzzentrum für Poliomyelitis erfolgen. Erst wenn 2 Kontrolluntersuchungen auf PV im Zeitintervall von einer Woche negativ ausfallen, dürfen Erkrankte bzw. Ausscheider wieder Gemeinschaftseinrichtungen besuchen. Kontaktpersonen sollten umgehend eine IPV verabreicht werden $[7,11,13]$.

\section{Arbeitsmedizinische Aspekte}

Unter bestimmten Voraussetzungen könnten bestimmte Berufszweige einem hohen Infektionsrisiko ausgesetzt sein $[7,11,13]$ :

1. Angestellte in Gemeinschaftseinrichtungen für Emigranten, Flüchtlinge und Asylbewerber aus Gegenden mit PM-Prävalenz,

2. Mitarbeiter aus dem medizinischen Bereich mit Kontakt zu PM-Erkrankten,

3. Mitarbeiter in Laboratorien mit PMGefahr.

Besonders die oben genannten Personen sind durch prophylaktische Maßnahmen wie Impfungen oder Einhaltung der Hygienevorschriften vor einer potenziellen Infektion zu schützen. Des Weiteren sollten Aufklärungen bezüglich des Infektionsweges, der Symptomatik und der Prävention bei einer Infektion erfolgen, sodass im Krankheitsfall eine schnelle Umsetzung des nötigen Verfahrensweges erfolgen kann. Für Personen mit einem Infektionsrisiko für PM am Arbeitsplatz sind regelmäßige arbeitsmedizinische Vorsorgen nach der ArbMedVV vorgesehen [3, 7, 11, 13].

\section{Reisemedizinische Aspekte}

Die WHO hat aufgrund der internationalen Ausbreitung der Poliomyelitis am 5. Mai2014 eine „Public Health Emergency of International Concern (PHEIC)“ erklärt, informiert das RKI im Epidemiologischen Bulletin 19/2014. Für Personen, die einen Aufenthalt von mehr als 4 Wochen in einem der betroffenen Länder planen, kann eine vorgezogene Auffrischimpfung gegen Polio sinnvoll sein [16].

\section{Fazit für die Praxis}

- Bei der PM handelt es sich um eine akute virale Infektionskrankheit des ZNS, die durch das PV verursacht wird.

- Primär wird die Krankheit durch engen Kontakt mit einer infizierten Person übertragen, wobei ein fäkal- 
oraler oder ein aerogener Übertragungsweg möglich ist.

- Die Inkubationszeit beträgt 2-35 Tage.

- In den meisten Fällen verläuft die Infektion asymptomatisch, jedoch kommt es bei $5 \%$ der Patienten zu einer klinischen Symptomatik.

- Die Behandlung erfolgt symptomatisch mittels Physiotherapie und orthopädischer Hilfsmittel.

- Die Impfung sollte durch den inaktiven Impfstoff (IPV) bei Kindern im Rahmen der Grundimmunisierung erfolgen.

- In Entwicklungsländern kommt noch das orale Poliovakzin (OPV) zum Einsatz, das jedoch seit 1998 in Deutschland aufgrund möglicher unerwünschter Ereignisse nicht mehr empfohlen wird.

- Insbesondere Personen, die einem Infektionsrisiko ausgesetzt sind, sollten über alle Aspekte der PM aufgeklärt werden.

\section{Korrespondenzadresse}

\section{Dr. N. Schöffel}

Institut für Arbeitsmedizin, Sozialmedizin und Umweltmedizin, Goethe-Universität Frankfurt Theodor-Stern-Kai 7, 60590 Frankfurt am Main, Deutschland

Schoeffel@med.uni-frankfurt.de

Interessenkonflikt. D. N. Schöffel, U. Drews, M. H. K. Bendels, D. Brüggmann und D. A. Groneberg geben an, dass kein Interessenkonflikt besteht.

\section{Literatur}

1. Baj A, Colombo M, Headley Jl et al (2015) Postpoliomyelitis syndrome as a possible viral disease. Int JInfect Dis 35:107-116

2. Franck S, Allwinn R, Rabenau HF et al (1999) Epidemiological analysis of immunity to poliovirus after termination of an era of vaccination with OPV in Germany. An analysis of the German Association Against Viral Diseases (DVV). Zentralbl Bakteriol 289:475-481

3. Garon JR, Cochi SI, Orenstein WA (2015) The challenge of global poliomyelitis eradication. Infect Dis Clin North Am 29:651-665

4. Goncalves G, Santos LA, Sarmento A et al (2003) Portugal and Europe are poliomyelitis free. Acta MedPort 16:33-39

5. Kozic D, Turkulov V, Bjelan M et al (2014) Extensive myelitis after oral polio vaccination: MRI features. Jbr-Btr 97:358-360

6. Loucq C (2013) Vaccines today, vaccines tomorrow: a perspective. Clin Exp Vaccine Res 2:4-7
7. Mehndiratta MM, Mehndiratta P, Pande R (2014) Poliomyelitis: historical facts, epidemiology, and current challenges in eradication. Neurohospitalist 4:223-229

8. Platt LR, Estivariz CF, Sutter RW (2014) Vaccineassociated paralytic poliomyelitis: a review of the epidemiology and estimation of the global burden. J Infect Dis 210(Suppl 1):S380-S389

9. Santos AP, Costa EV, Oliveira SS et al (2002) RTPCR based analysis of cell culture negative stools samples from poliomyelitis suspected cases. J Clin Virol 23:149-152

10. Strauss R, Sagl M, Wewalka G et al (2008) WHO Polio Eradication Programme:Status quo and implementation in Austria. Wien Klin Wochenschr 120:210-216

11. Tajaldin B, AlmilajiK, Langton Petal (2015) Defining polio: closing the gap in global surveillance. Ann Glob Health 81:386-395

12. Trallero G, Cabrerizo M, Avellon A (2013) Role of the National Poliovirus Laboratory for the program of eradication and poliomyelitis surveillance. Rev Esp Salud Publica 87:471-479

13. Wiese-Posselt M, Tertilt C, Zepp F (2011) Vaccination recommendations for Germany. Dtsch Arzteb Int 108:771-779 (quiz 780)

14. Wilton T, Dunn G, Eastwood D et al (2014) Effect of formaldehyde inactivation on poliovirus. J Virol 88:11955-11964

15. Mitteilung der Ständigen Impfkommission am Robert Koch-Institut (RKI, 2015): Empfehlungen der Ständigen Impfkommission (STIKO) am Robert Koch-Institut. http://www.rki.de/DE/ Content/Infekt/EpidBull/Archiv/2015/Ausgaben/ 34_15.pdf?_blob=publicationFile. Zugegriffen: 20.10. 2015

16. Polio:Weltgesundheitsorganisation (WHO) erklärt Polio-Ausbreitung zur "Gesundheitlichen Notlage mit internationaler Tragweite". http://www.rki.de/ DE/Content/Infekt/EpidBull/Archiv/2014/19/Art 02.html;jsessionid $=5$ C5055655003FD672C2A 96762604F62B.2_cid290?nn=2375548. Zugegriffen:31.11.2015

\section{September 2016}

Berlin 01.-02.09.2016

Der Demografiekongress 2016

Zukunftsforum Langes Leben

Themen: Wohnen und Dienstleistungen, Arbeits-

welt und Personal, die kommunale Gestaltung

des demografischen Wandels

sowie Pflege und Medizin

Auskunft: Gesundheitsstadt Berlin,

Schützenstraße 6A, 10117 Berlin,

Fon: (030) $7001176-00$

office@gesundheitsstadt-berlin.de,

http://www.gesundheitsstadt-berlin.de/

Wuppertal 19.-21.09.2016

Internationale, interdisziplinäre ICOH-

Konferenz,,Work, Age, Health and

Employment-Evidence from Longitudinal

Studies (WAHE2016)"

Themen: Arbeit und Berentung; Arbeit, Alter

und Gesundheit; Lebenslaufperspektive in der

Renteneintrittsforschung;Veränderungsmessung

in Längsschnittstudien; Länderübergreifende

Forschung - Chancen und Herausforderungen

Wiss. Leitung: Prof. Dr. H. M. Hasselhorn,

Dr. J.-B. du Prel

Auskunft: Lehrstuhl für Arbeitswissen-

schaft, Fakultät für Maschinenbau und

Sicherheitstechnik

Bergische Universität Wuppertal,

Gaußstr. 20, 42119 Wuppertal,

Fon: +49 202 439-3008/3009

wahe2016@uni-wuppertal.de,

www.wahe2016.uni-wuppertal.de/

\section{Oktober 2016}

Bamberg 22.10.2016

\section{Die Wirbelsäule}

15. Orthopädisch- Unfallchirurgisches Symposium

Themen: Konservative und operative Behandlung

von Wirbelsäulenerkrankungen, Begutachtung

Wiss. Leitung: Prof. Dr. Peter Strohm

Dr. Wolfgang Willauschus

Auskunft: Frau Gudrun Willauschus, wicoMED,

Georg-Hofmann-Str. 12, 95488 Eckersdorf,

Fon: 0172-7209852

info@wicomed.de,

www.wicomed.de

Berlin 25.-28.10.2016

Deutscher Kongress für Orthopädie und

Unfallchirurgie - DKOU 2016

Auskunft: Intercongress $\mathrm{GmbH}$

Wilhelmstraße 7, 65185 Wiesbaden

Fon: 0611/977160,

dkou@intercongress.de,

www.dkou.de

Dresden 26.-29.10.2016

Deutscher Betriebsärzte-Kongress 2016

32. Arbeitsmedizinische Jahrestagung des VDBW

Themen: Auswirkungen des Präventionsgesetzes

für Betriebsärzte, Arbeitsmedizinische Regeln

und Empfehlungen, Arbeitsmedizin - aus der

Vergangenheit für die Zukunft, Psychische

Gesundheit im Betrieb

Wiss. Leitung: Dr. Uwe Gerecke und Dr. Martin Kern

Auskunft: Verband Deutscher Betriebs- und

Werksärzte e. V.,

Friedrich-Eberle-Straße 4a, 76227 Karlsruhe,

Fon: 0721 933818-0,

info@vdbw.de,

www.vdbw.de 